\title{
Dependence of SLS Parameters on Thermal Properties of Composite Material of Cement with Polyamide 12
}

\section{SA Aldahsh*}

The Mechanical Engineering Department, Riyadh College of Technology, Saudi Arabia

\begin{abstract}
SLS employs powder-processing in the construction of parts and SLS parameters depending on the thermal properties of the powder. An average of $80 \%$ to $90 \%$ of the powder in the build chamber is not sintered during the SLS process and could be reused in relation to its properties. However, the properties of un-sintered powder deteriorate due to exposure to a various temperature for extended periods of time during the SLS system material build-process in three stages, starting from warm up stage, the build stage, where the powder is exposed to just below the melting point of the material, and the cool-down stage. An experimental study of the thermal properties of composite material of cement and PA12 to determine the optimum parameters of SLS process are being investigated. The investigation uses the thermal properties of different proportions of composite material of cement and PA12. In addition, an experimental study of the thermal properties and physical properties of used or un-sintering powder to expand a methodology of controlling the SLS parameters, in turn to obtain consistent, good quality fabricated SLS specimens.
\end{abstract}

Keywords: Rapid Prototype (RP); Selective Laser Sintering (SLS); Polyamide 12; Portland cement

\section{Introduction}

One of the most common of Rapid Prototyping (RP) techniques exist is Selective Laser Sintering (SLS) which is capable of producing very complex part-geometry directly from three-dimensional CAD software. SLS employs powder-processing in the construction of parts. Sintered parts are fabricated when the surface tension of particles is overcome in the heat of an infrared laser beam and they subsequently fuse together. The powder is supplied by two feed-cartridges which distribute a thin layer of powder over the build-area by a rotating roller so that the next layer of powder may be added, and the process continues in this way until the part is completed [1].

The SLS process starts by transferring CAD data files in STL file format to the SLS machine where they are sliced. Build parameters and guidance of the laser are controlled by the data which is processed by a computer system. As well as this, the computer system is used to control nitrogen which creates an inert atmosphere to eliminate the possibility of powder oxidation and explosion. This is followed by heating the powder to a temperature just below the melting point of the material using a heater which is located above the part-bed, in order to minimize thermal distortion and heat stress to the lowest possible degree; prevent the fabrication part from warping and facilitate fusion to the previous layer. The temperature of the powder-feed cartridges is controlled to allow powder to be moved freely by a rotating roller. A very thin layer (between $100 \mu \mathrm{m}$ to $125 \mu \mathrm{m}$ ) is spread by the roller across the part build. The speed of the roller transverse is an adjustable machine parameter $[2,3]$.

This paper presents a theoretical and experimental study of composite material of cement and PA12 powder properties as the thermal, the molecular weight, additives, properties of different proportion of composite material of cement and PA12 of the powder. The experiment study deals the effect of the cement proportion on viscosity, melts flow rate, thermal behavior and molecular weight of polyamide12, the effect of the temperature and time on the molecular weight, the effect of the temperature and time on the viscosity and melts flow rate, the effect of continuous and cyclic heat on MFR and molecular weight and the effect of the temperature and time on $\mathrm{Tg}$,
Tm and Tc, in order to obtain consistent and have good quality of the fabricated SLS specimens.

Most SLS technologies can be classified using possible classification of SLS technologies according to the binding mechanism. The SLS of material reinforced polymers can be seen as liquid phase sintering of composite powder particles, where the polymer is a binder and the material reinforced particles represent a structural material. The polymer does not just act as a binder material but constitutes the main phase of the structural material, and basically a structural material that is fully molten, while the material reinforced particles remain solid [4].

\section{Thermal properties of the powder}

In the SLS process, where the fabrication parameters involve using composite powder as a reinforced-polymer powder, the polymer acts as a binder within the reinforced powder, so the fabrication parameters are mainly dependent on the properties of polymers. As shown in Figure 1 , the most important characteristics determining the application of thermoplastic polymers are Glass Transition Temperature Tg, Melting Temperature Tm and Crystallization Temperature Tc $[5,6]$.

\section{Glass transition temperature $T g$}

The Glass Transition Temperature, $T g$ is the temperature where a rapid decrease in elastic modulus occurs. It varies with different polymers and takes place in an amorphous region of the semicrystalline polymers. At ambient temperature, the amorphous regions are solid and the polymer is in a glassy state which causes it to be very hard and brittle. $\mathrm{Tg}$ occurs due to an increase in the motion of large

${ }^{*}$ Corresponding author: S.A. Aldahsh, Mechanical Engineering Department, Riyadh College of Technology, Saudi Arabia, Tel: 96614670000; Fax: 96614677580; E-mail: esad353@hotmail.com

Received September 03, 2013; Accepted November 26, 2013; Published December 05, 2013

Citation: Aldahsh SA (2013) Dependence of SLS Parameters on Thermal Properties of Composite Material of Cement with Polyamide 12. J Appl Mech Eng 2: 130. doi:10.4172/2168-9873.1000130

Copyright: (C) 2013 Aldahsh SA. This is an open-access article distributed under the terms of the Creative Commons Attribution License, which permits unrestricted use, distribution, and reproduction in any medium, provided the original author and source are credited. 
segments of molecular chains caused by heating from below $\mathrm{Tg}$. When a certain amount of heat is applied, there is a point where the behaviour of the polymer rapidly changes due to breaks in the van der Waal bonds. At this point, the mechanical behaviour of the polymer changes from glassy and brittle and becomes less rigid and rubbery. However the crystalline regions are maintained, due to stronger covalence bonds, so melting does not occur until a higher temperature is applied [5-8].

There are a number of structural features which have a bearing on the value of glass transition temperature. As $\mathrm{Tg}$ depends largely on the amount of thermal energy required to keep the polymer chains moving, a number of factors influence $\mathrm{Tg}$, including chain flexibility, molecular structure, molecular weight, branching and cross-linking. The flexible chain has a low $\mathrm{Tg}$ whereas a rigid chain has a high $\mathrm{Tg}$. Molecular structure and the size of the group attached to the main chain carbon atom can influence the glass transition point. In this case, the steric factor affects the flexibility of the chain and these arise when bulky pendant groups hinder the rotation around the backbone and cause $\mathrm{Tg}$ to increase. The molecular weight of a polymer will have some effect on the $T g$, so an increase in molecular weight causes an increase in the glass transition temperature. Cross-links will also have an impact on the $\mathrm{Tg}$ when they are introduced into a polymer. The density of the sample is increased proportionally and this leads to a restriction of the molecular motion in the sample and a rise in $\operatorname{Tg}[5,9]$

\section{Melting temperature Tm}

The melting temperature is the temperature where the polymers eventually obtain sufficient thermal energy as the temperature rises to enable its chains to move freely enough and to behave like a viscous liquid. The melting temperature is typically in the order of $100^{\circ} \mathrm{C}$ above the glass transition temperature, $\mathrm{Tg}$, or $50^{\circ} \mathrm{C}$ above Crystalline Temperature, $T c$. This varies for different polymers [5]. When the temperature rises from $\mathrm{Tg}$ to about $30 \mathrm{~K}$ above $\mathrm{Tg}$, the molecular motion starts to increase and this leads the modulus of elasticity to go down, with continuance in temperature rising to just above Tm, where the polymer becomes liquid and the chains are all tangled up with their neighbours [7]. The viscosity of the molten polymer depends significantly on the temperature; as the temperature increases, the viscosity of the polymer decreases considerably and can be expressed in the form $[5,10,11]$ :

$$
\eta_{0}=A \exp ^{(\Delta E / R T)}
$$

where $\Delta E$ is the activation energy for viscous flow, $\eta_{0}$ the viscosity, $A$, a constant specific to molecules mobility, $R$, the gas constant, and $T$, the absolute temperature.

As the Melting Temperature, Tm, depends largely on the amount of thermal energy required to keep the polymer chains moving, a number of factors influence Tm, i.e. symmetry, intermolecular bonding, branching and molar mass. The symmetry of the chain shape influences the Tm. More thermal energy is required for polymers that are sufficiently symmetrical to be considered as smooth, stiff cylindrical rods and this leads to raised $\mathrm{Tm}$. The intermolecular bonding will affect the melting temperature, so any interaction between chains in the crystal lattice will help to hold the structure together more firmly and raise the melting temperature. The branching in the side group tends to stiffen the chain and raise the melting temperature. The last factor is molar mass which can change Tm. This is where, if the molar mass in the sample increases, the melting temperature will rise $[5,12,9,11]$.

\section{Crystallisation temperature $T c$}

The crystallisation temperature occurs between Melting
Temperature $\mathrm{Tm}$ and glass transition temperature $\mathrm{Tg}$. Usually, the rate of crystallisation is seen between $10 \mathrm{~K}$ below melting point and $30 \mathrm{~K}$ above glass transition temperature [5]. At temperatures close to melting point, the crystallisation rate is low and large crystal sizes can be obtained, while at temperatures far below melting point and near $\mathrm{Tg}$, the melt is so viscous that molecular motion is extremely slow, thus leading to a faster crystallisation rate.

The maximum rate of crystallisation takes place at Tc (Figure 2) [12] and this is influenced by molecular mass. The sample with the lower molecular mass crystallises faster. In addition to this, the presence of homogeneous or heterogeneous nuclei affects the overall crystallisation rate by facilitating the initial nucleation step. The reason for this is that even in the melts; some hydrogen bonds remain fixed and result in effective nucleation sites on cooling. The thermal history should therefore be erased and all homogeneous nuclei destroyed by holding the product for a sufficient time in the melt before crystallisation kinetics can take place $[5,12,9]$.

The growth of a crystal nucleus can take shape in either one, two or three dimensions with the crystals in the form of rods, discs or spheres respectively. The crystal growth rate is strongly dependent upon the crystallisation temperature. When the Crystallisation Temperature Tc remains just below the melting temperature of the polymer the growth rate is relatively low. As mentioned early in Equation (1), when the temperature is reduced, there will be an increase in viscosity and

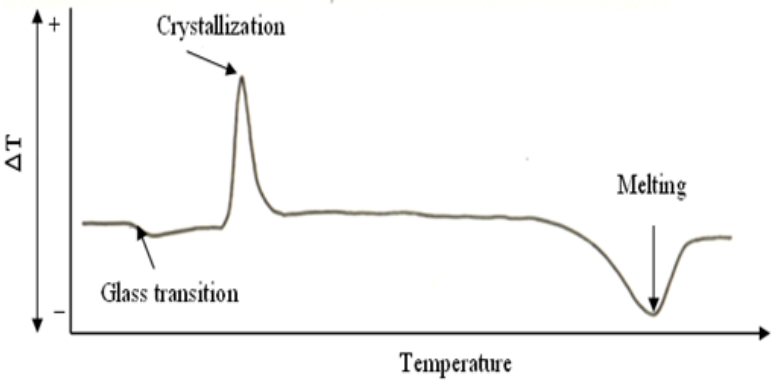

Figure 1: Characteristics that determine the application of thermoplastic polymers (Cowie, 1973).

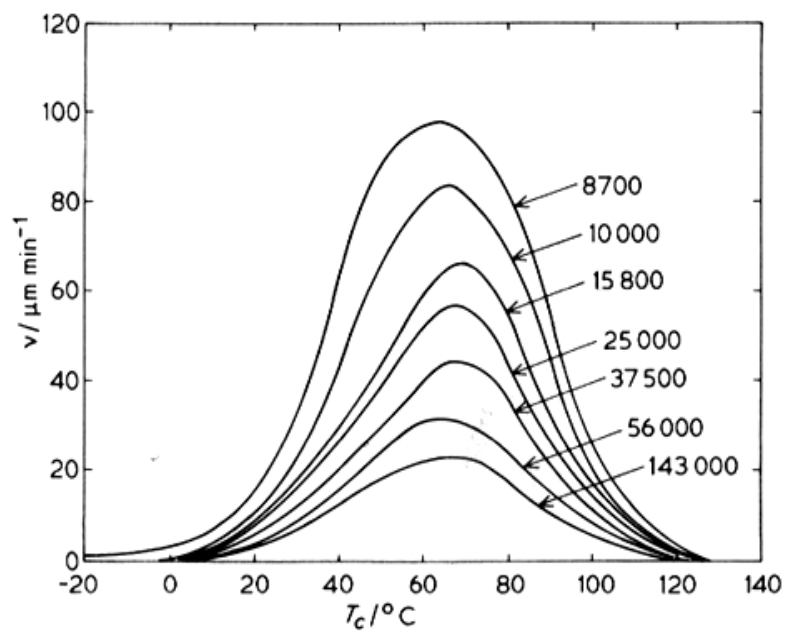

Figure 2: Crystallisation Temperature $T c$ with crystal growth rate $v$ for different molar mass in $\mathrm{g} \mathrm{mol}^{-1}$ (Young, 1981) 
transport of material to the growth point will be more difficult. Growth rate therefore peaks, and as the temperature decreases to close to glass transition temperature, the growth rate decreases. The growth rate also depends upon the molar mass $\mathrm{M}$. As $\mathrm{M}$ is reduced, the growth rate increases (Figure 2) [12].

\section{Molecular weight}

The most important property for describing the characteristic of polymers is molecular weight, which influences the thermal properties of polymers through the melting viscosity. This is due to the fact that high molecular weight can lead to difficulties in forming the material given the high viscosities involved. The general effects of increasing molecular weight are seen to be that molecular weights fall below a critical value $M c$ from 5000-1500. The viscosity is directly proportional to the average molecular weight, but above $M c$ viscosity, they depend on a greater power (Figure 3) $[5,10,11]$.It has been found that the melting viscosity varies largely with changes in the molecular weight for many polymers, as shown in the equation:

$$
\eta_{0}=\mathrm{K}\left(\mathrm{M}_{\mathrm{w}}\right)^{\mathrm{n}}
$$

where $\eta_{0}$ is viscosity and Mw is molecular weight, $\mathrm{K}$ is a constant that depend upon the type of polymer and exponent $n=3.4-3.5$.

\section{Additives}

Additives are commonly used in nylon and these include reinforcements, such as ceramic and glass fibers. Reinforcements play an essential role in composite material. They can produce a number of attractive effects in the mechanical properties of specimens such as increased flexural strength, compression, stiffness, improvement in fracture toughness, creep resistance and, in some cases, tensile strength. To be a candidate for reinforcing nylon, a product must have a melting point above that of nylon [9]. Additives of filler to polyamide directly affect viscosity. As the fillers increase, the viscosities of the composite material increases proportionally (Figure 4). The Einstein equation declares that the filler increases viscosity in a consistent manner,

$$
\eta_{c} / \eta_{0}=1+2.5 \phi
$$

While Guth equation declares a simple extension of Einstein's relationship to higher concentration [9].

$$
\eta_{c} / \eta_{0}=1+2.5 \phi+14.1 \phi^{2}
$$

where $\eta_{c}$ is the viscosity of the filled polymer at the volume

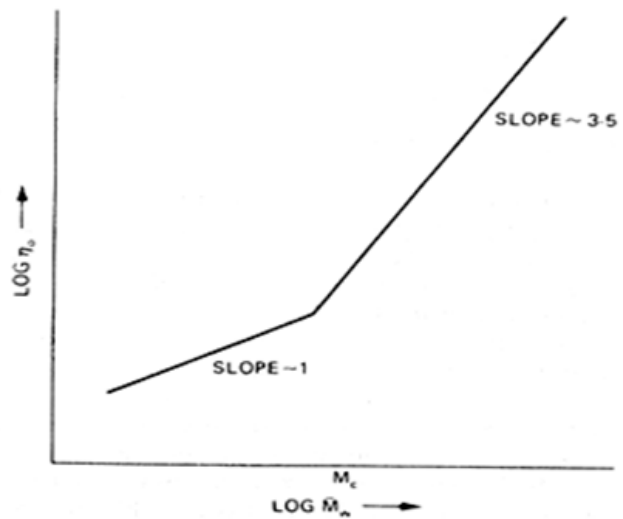

Figure 3: Relationship between molecular weight $M w$ and viscosity $\eta_{0}$ (Brydson, 1999).

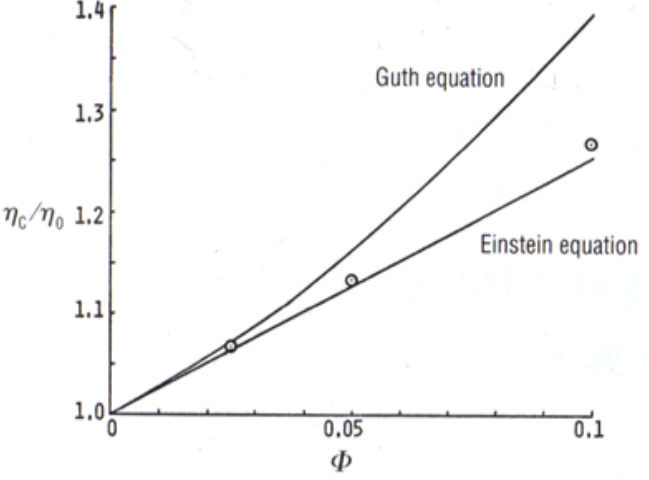

Figure 4: Relative Viscosities with volume fraction filler (Kohan, 1995).

concentration $\phi, \eta_{0}$ is the zero shear rate melt viscosity of the unfilled polymer.

\section{Experimental Work}

\section{Methodology}

In order to simulate the conditions of the SLS process, the samples were set in the Oven Heraeus Instruments Vacutherm, Type 6060M, in a maximum amount of nitrogen atmosphere to prevent the powders from oxidizing (yellowing) and heated at a specified temperature for varying periods. The samples were heated at temperatures $100^{\circ} \mathrm{C}$ and $180^{\circ} \mathrm{C}$, the reason being that $100^{\circ} \mathrm{C}$ is considered the minimum degree to which the powder would be exposed through the SLS process, located in the left and right cartridges, while $180^{\circ} \mathrm{C}$ is considered the maximum degree to which the powder would be exposed through the SLS process, located in the bed part. The samples were kept for 20, 40, 60,80 , and 100 hours at each temperature. They were heated using two methods, continuous heating and cyclic heating. The first method was used to simulate the SLS process for one build and the second method for simulating the SLS process for a number of builds by taking out the samples from the oven to cool down until room temperature before returning them to the oven. Samples of new and recycled grade powder were tested, and the results analysed to investigate the effect of the temperature, time and number of builds on the powder properties.

\section{Results and Discussion}

The effect of the cement proportion on viscosity, melts flow rate, thermal behaviour and molecular weight of polyamide12

Graphs relating to viscosity, melt flow rate, thermal behaviour and molecular weight values of cement/PA12 composite in relation to various proportions of cement are shown in Figure 5. As mentioned earlier in Section 2.3, theoretically according to the equations number 3 and 4, the addition of filler to polyamide directly theoretically affects viscosity. As the fillers increase, the viscosity of the composite material increases proportionally. In this experiment, viscosity and melt flow rate of cement/PA12 composite is affected via different proportions of cement added to Polyamide 12. Figure 5a and 5b show that average, minimum and maximum results were tested using the Melt Flow Rate Indexer. From Figure 5a, the relationship between the viscosity of the composite and weight fraction (wt\%) of the cement can be observed. It can be seen that the viscosity increases gradually with an increase in the weight fraction (wt\%) of the cement. From the results obtained, this suggests that the cement particles can affect the melt viscosity of 
(A)

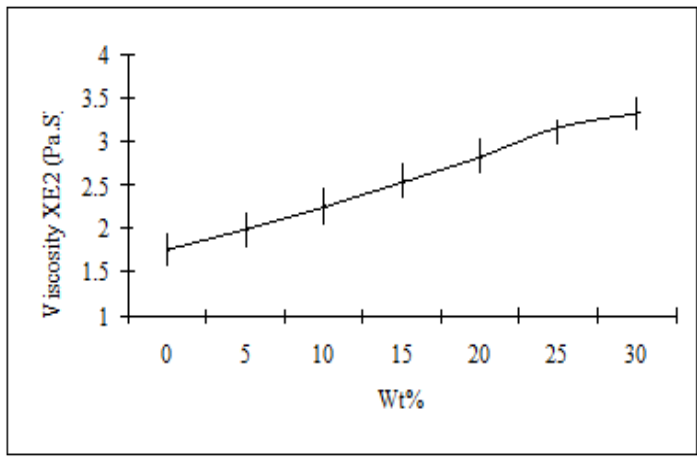

(B)

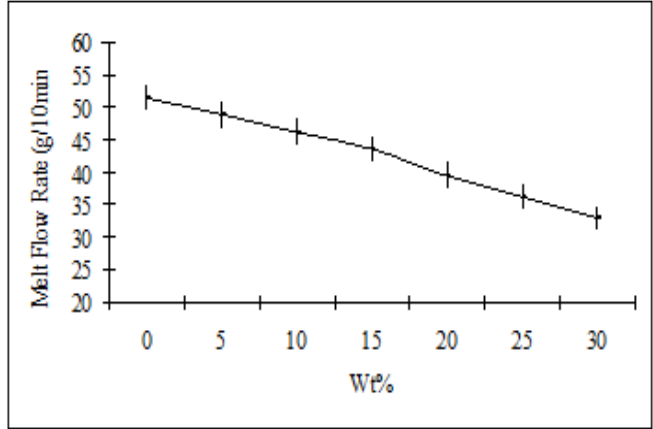

(C)

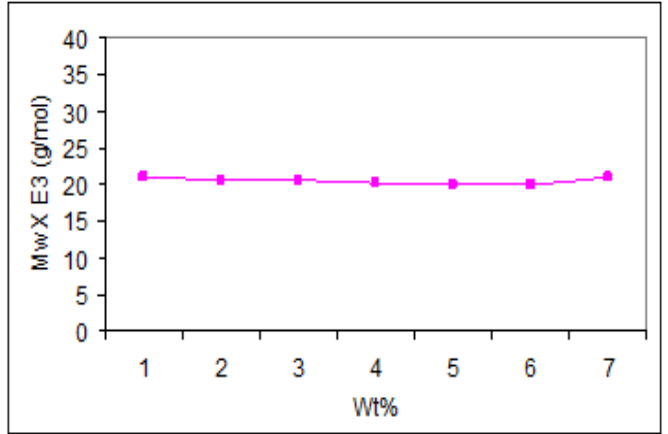

(D)

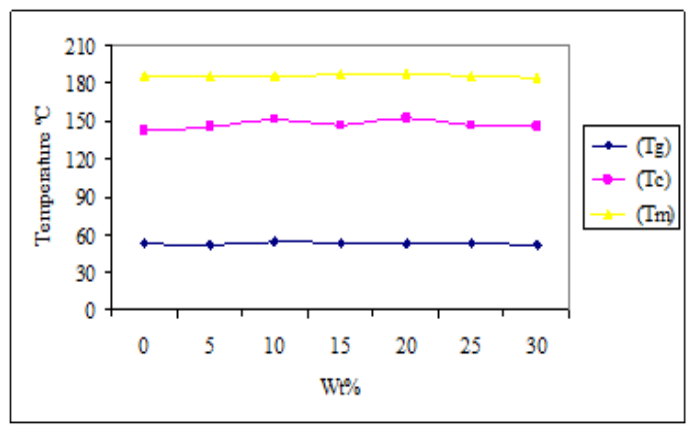

Figure 5: Variation of (a) viscosity, (b) MFR, (c) molecular weight and (d) $\mathrm{Tg}, \mathrm{Tm}$ and Tc with cement content add to polyamide12.

the composite. This means that addition of rigid particles like cement to Polyamide 12 can increase viscosity due to the decrease in melted Polyamide 12 and the fact that the gap between the cement particles becomes narrower. This leads to stickier extruded material.

Figure $5 \mathrm{~b}$ demonstrates a melt flow rate which has decreased at the same time the weight fraction (wt\%) of the cement has increased. Therefore, it is clear from an examination of the data presented that the weight fraction (wt\%) of the cement used has had an influence on the MFR value, and this is due to an increase in the viscosity of the composite as the filler increases. As mentioned earlier, the MFR depends on the mass and time-interval cut-off of the extruded material. Therefore, when the viscosity increases, this leads to an influence on mass and the time frame of the extruded material, ultimately causing the MFR of the composite to decrease.

Figure $5 \mathrm{c}$ and $5 \mathrm{~d}$ show the results were tested using Gel Permeation Chromatography (GPC) and DSC analysis, respectively. These figures illustrate the relationship between the molecular weight and $\mathrm{Tm}, \mathrm{Tg}$ and Tc of the composite in relation to various proportions of cement. In this experiment, the molecular weight and $\mathrm{Tm}, \mathrm{Tg}$ and $\mathrm{Tc}$ of cement/ PA12 composite are not affected via different proportions of cement added to the Polyamide 12. As the $T g$ and $\mathrm{Tm}\urcorner$ are used to calculate the part bed temperature, the results obtained suggest that the part bed temperature is fixed by different proportions of cement added to the Polyamide 12 .

The Effect of the Temperature and Time on the Molecular Weight: In this experiment, the composite material of cement/PA12 was exposed to $100^{\circ} \mathrm{C}$ and $180^{\circ} \mathrm{C}$ temperatures in an oven with a nitrogen atmosphere. The purpose was to simulate the deterioration of the composite powder during the SLS process and after that, arrive at the maximum proportion of used powder that can be re-used in the SLS process.

Figure 6 show the results were tested using Gel Permeation Chromatography (GPC). This figure illustrates the relationship between the Molecular Weight (Mw) and the heating period. It can be seen from the figure that the powder heated to $100^{\circ} \mathrm{C}$ (considered to be the minimum degree the powder would be exposed to during the SLS process) has a lower molecular weight and experiences a leisurely increase compared to the powder heated at $180^{\circ} \mathrm{C}$. For instance, the fastest change in molecular weight takes place at $180^{\circ} \mathrm{C}$. Temperature plays an essential role in the Mw, as shown in Figure 6. Mw at the high temperature of $180^{\circ} \mathrm{C}$ increases rapidly for the first 25 hours from 20 $\mathrm{E} 3 \mathrm{~g} / \mathrm{mol}$ to $141 \mathrm{E} 3 \mathrm{~g} / \mathrm{mol}$. After a 25 hour heating period, the increase in $\mathrm{Mw}$ is slower. From this figure, it can be seen that temperature has a more significant influence on the Mw of the powder than SLS time. It is therefore $\mathrm{Mw}$ which affects the quality of SLS parts.

The Effect of the Temperature and Time on the Viscosity and Melts Flow Rate: As mentioned earlier in Section 2.2, Equation 2 theoretically demonstrates that the viscosity of polyamide is affected by molecular weight. As molecular weight depends on exposure of the powder to various temperatures, the viscosity of the powder directly increases (Figure 7). In this experiment, the composite material of cement/PA12 has been exposed to temperatures as described in Section 3.1 and the results are presented in Figures 8 and 9.

Figures 8 and 9 demonstrate the results of viscosity and MFR, respectively, of the composite material of cement/PA12. These have been through varying temperatures and timings, and were tested using the Melt Flow Rate Indexer. From these figures, it is clear that temperature and heating time play an essential role in the viscosity and 


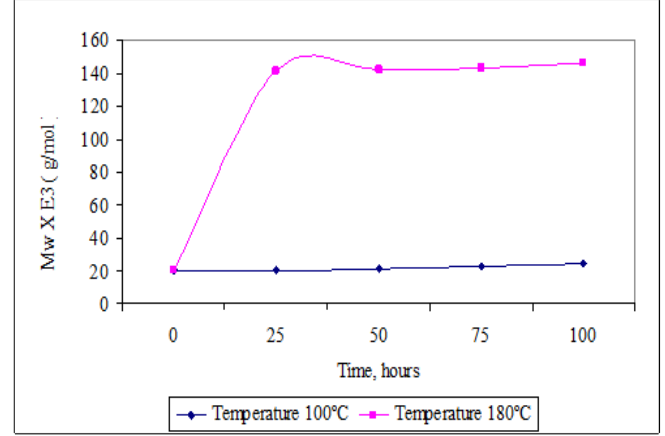

Figure 6: Variation of molecular weight $(\mathrm{Mw})$ of composite of cement/polyamide12 powder with different temperature and time.

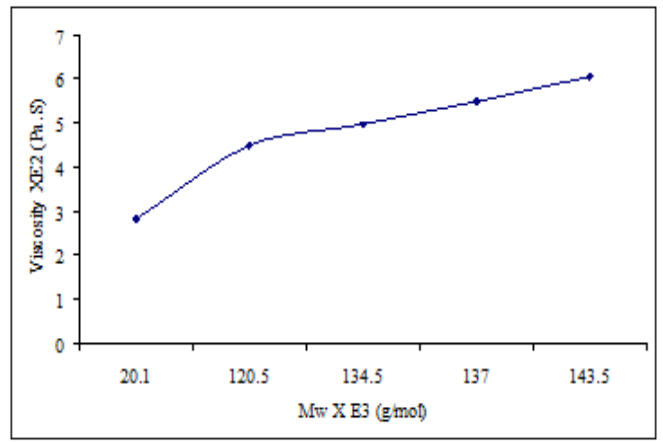

Figure 7: Relationship between molecular weight $\mathrm{Mw}$ and viscosity $\eta_{0}$

MFR of the composite material. The largest increase in viscosity appears to be at $180^{\circ} \mathrm{C}$ in the first 25 hours, so it is clear from Figure 8 there is a rapid increase in viscosity at $180^{\circ} \mathrm{C}$ during the first 25 hours, and then a gradual increase after that period. In contrast, at the same temperature of $180^{\circ} \mathrm{C}$ and during the same time period, the largest decrease in MFR occurred (Figure 9). This was attributed to increased molecular weight, thus leading to an increase in viscosity, followed by decreasing MFR. New powder unexposed to temperature has the lowest viscosity and highest MFR, while powder exposed to the highest temperatures and longest time periods has the highest viscosity and lowest MFR.

The Effect of Continuous and Cyclic Heat on MFR and Molecular Weight: In the following experiments, the samples of composite material of cemment/PA12 were exposed at temperature $180^{\circ} \mathrm{C}$ in an oven with a nitrogen atmosphere, and method of heating referred to as continuous and cyclic heating. In the method of cyclic heating the powder samples were placed in the oven at temperature $180^{\circ} \mathrm{C}$ and heated for 25 hours. Then they were taken out and cooled down to room temperature. After taken a little amount of the powder to measure and record their MFR and molecular weight, they were returned back into the oven for another 25 hours. This procedure was repeated in 25 hour periods for up to 100 hours in total. The results of these experiments are shown in a bar chart format in Figures 10 and 11. The purpose is to explore whether the cyclic heating would have a different effect on composite powder deterioration than the LS time alone.

Figures 10 and 11 show molecular weight and MFR of composite material of cemment/PA12 respectively obtained as opposed to heating period at two methods, continuous and cyclic heating. These results show a clear trend of a slightly higher deterioration rate of the samples subjected to continuous heating against those subjected to cyclic heating. Generally, this difference between these methods is not very significant for effect on molecular weight and MFR as influence of temperature and heating period on material deterioration.

The Effect of the Temperature and Time on $\mathrm{Tg}$, Tm and Tc: Figures 12-14 show that the results were tested using DSC analysis. These figures illustrate the relationship between $\mathrm{Tg}, \mathrm{Tm}$ and $\mathrm{Tc}$ of the composite material of cement/PA12 and various temperatures and heating period. In this experiment, the samples of composite material have been exposed to the temperatures described in Section 3.1. As mentioned earlier in Section 2.1, the common factor affecting the three Transition Temperatures $\mathrm{Tg}, \mathrm{Tm}$ and $\mathrm{Tc}$ is theoretically speaking, molecular weight. As the molecular weight increases, the $\mathrm{Tg}$ and $\mathrm{Tm}$ of the material increase, while $T c$ of the material decrease.

Figures 12 and 13 demonstrate that the powder heated to a temperature of $100^{\circ} \mathrm{C}$, which is considered to be the minimum degree to which the powder would be exposed during the SLS process, has lower $\mathrm{Tg}$ and $\mathrm{Tm}$ experiences a leisurely increase compared to the powder heated at $180^{\circ} \mathrm{C}$. For instance, the fastest change in $\mathrm{Tm}$ takes place at $180^{\circ} \mathrm{C}$, so temperature clearly plays an essential role in the effect on thermal properties of composite material. As shown in Figures 12 and 13 , the $\mathrm{Tg}$ and $\mathrm{Tm}$ at the highest temperature of $180^{\circ} \mathrm{C}$ increase approximately by $5^{\circ} \mathrm{C}$ to $7^{\circ} \mathrm{C}$ within 100 hours. However, the $\mathrm{Tg}$ and $T m$ of the samples heated at the lowest temperature of $100^{\circ} \mathrm{C}$ increase by just $1^{\circ} \mathrm{C}$ to $3^{\circ} \mathrm{C}$ for the same period. In contrast, with regard to $T c$, as mentioned above, when the molecular weight increases, the $T c$ of the composite material decreases. It can be seen from Figure 14 that the

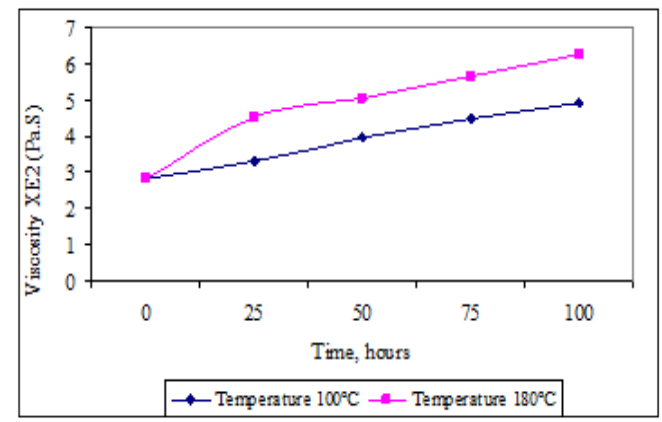

Figure 8: Variation of viscosity of composite of cement/polyamide12 powder with different temperature and time.

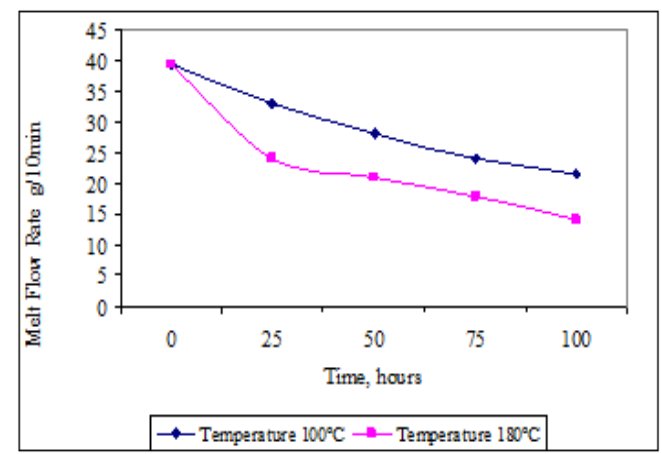

Figure 9: Variation of MFR of composite of cement/polyamide12 powder with different temperature and time. 


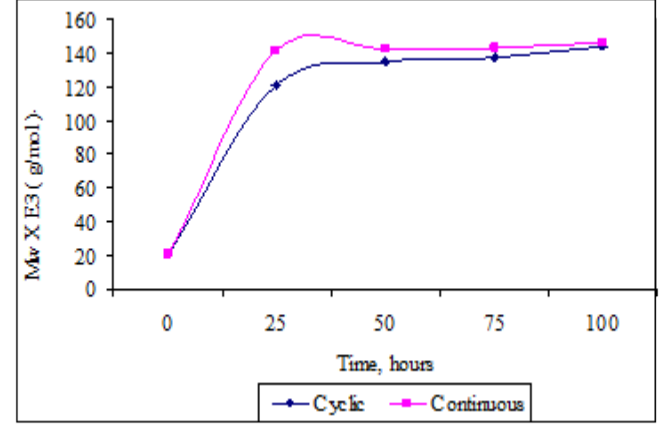

Figure 10: Effect of continuous and cyclic heat on Mw of composite of cement/ polyamide12 powder.

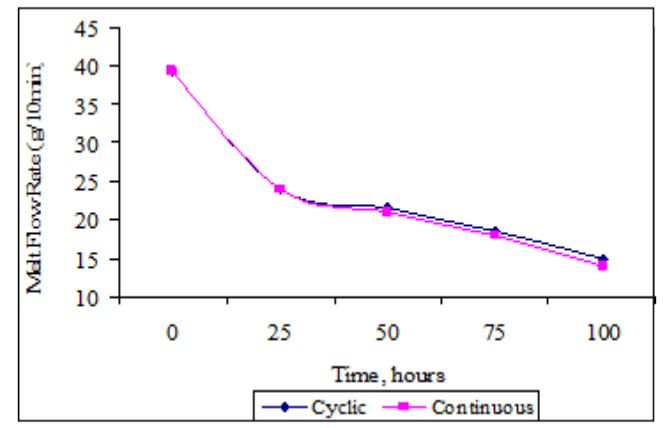

Figure 11: Effect of continuous and cyclic heat on MFR of composite of cement/polyamide12 powder.

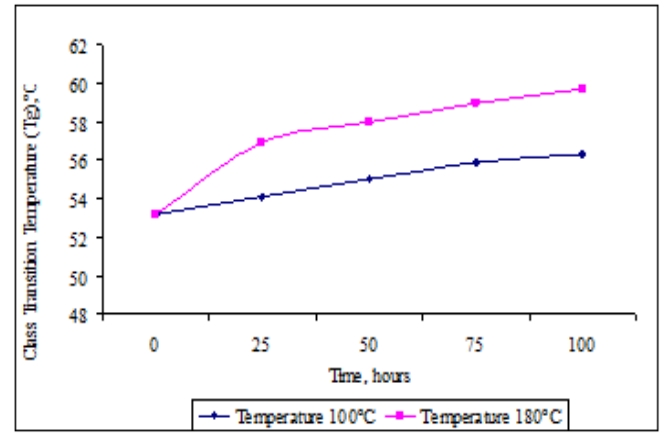

Figure 12: Effect of the temperature and time on $\mathrm{Tg}$ of composite of cement/ polyamide12 powder.

largest decrease in $T c$ occurs at $180^{\circ} \mathrm{C}$ and it is clear from Figure 14, there is a rapid decrease in $T c$ at $180^{\circ} \mathrm{C}$ and $100^{\circ} \mathrm{C}$ within the first 25 hours, and then a gradual decrease after that period. From these results, the $T g, T m$ and $T c$ are used to calculate the part bed temperature and thermal properties of the selected samples and these indicate that the thermal properties of the composite material of cement/PA12 change with reprocessing, thus affecting the properties of SLS parts.

\section{Discussion and Conclusions}

A better understanding of the thermal properties of composite material of cement/PA12 of both virgin powder, and used powder obtained from un-sintered powder is an enormous help in controlling SLS parameters and maintaining consistent quality of the fabricated SLS specimens.

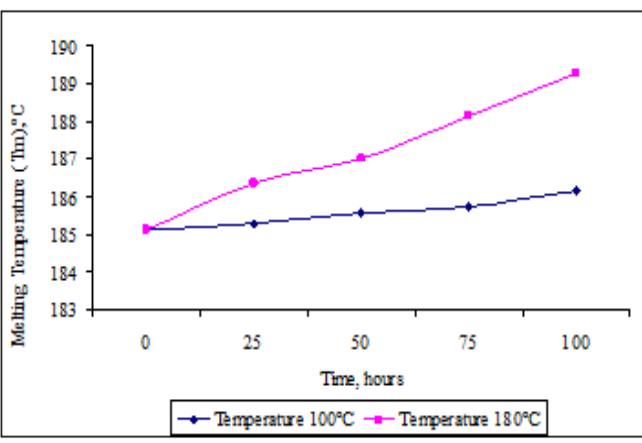

Figure 13: Effect of the temperature and time on Tm of composite of cement/ polyamide12 powder.

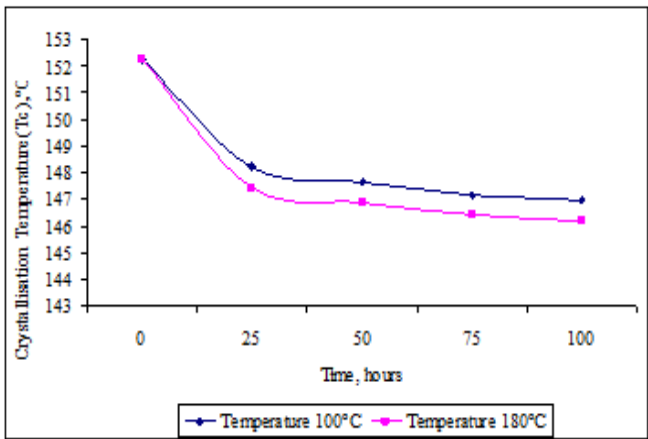

Figure 14: Effect of the temperature and time on Tc of composite of cement/ polyamide12 powder.

The combined indications of the differential scanning calorimeter suggest that the different proportions of cement added to the Polyamide 12 are not affected by the glass Transition Temperature $T g$, Melting Temperature $\mathrm{Tm}$ and Crystallisation Temperature $\mathrm{Tc}$. This can lead to fixing the part bed temperature with different proportions of cement added to the Polyamide 12, while the melt flow rate index test proposes that the addition of cement particles to Polyamide 12 affects the melt flow rate of the composite. As the proportion of cement increases, so does its viscosity, due to the decrease in melted Polyamide 12 and the fact that the gap between the cement particles becomes narrower. This leads to the extruded material becoming stickier, which is a sign of a decrease in the MFR index. It ultimately affects the quality and surface finish of the fabricated SLS specimens.

Huge amounts of powder left un-sintered during SLS could be reused depending on their properties. However, the properties of unsintered powder deteriorate due to exposure to high temperatures, e.g. just below the melting point of the composite material for an extended period of time during SLS building and the cool-down stage. The temperature and heating time to which the un-sintered powder are exposed are the most significant parameters for powder aging and deterioration of material properties.

The melt flow rate index tests indicate that the temperature to which the un-sintered composite powder was exposed and the duration of this exposure affect the melt flow rate of the composite. MFR of composite material decreases by $18-25 \mathrm{~g} / 10 \mathrm{~min}$ with powder usage and this was attributed to increased molecular weight, leading to increased viscosity and then decreased MFR. The powder left unexposed to such temperatures has the lowest viscosity and highest MFR, while the 
Citation: Aldahsh SA (2013) Dependence of SLS Parameters on Thermal Properties of Composite Material of Cement with Polyamide 12. J Appl Mech Eng 2: 130. doi:10.4172/2168-9873.1000130

Page 7 of 7

powder exposed to the highest temperature and longest period has the highest viscosity and lowest MFR. The molecular weight and melt flow rate of the samples subjected to continuous and cyclic heating are less significant than the temperature and heating time of the SLS process on material deterioration.

The differential scanning calorimeter DSC suggests that the temperature and heating time to which the un-sintered powder was exposed affect the Glass Transition Temperature Tg, Melting Temperature $\mathrm{Tm}$ and Crystallisation Temperature Tc. This leads to changes in part bed temperature with different grades of powder. The melting temperature of the composite material increases by $0.5-1{ }^{\circ} \mathrm{C}$ with each build or cycle until it reaches $4^{\circ} \mathrm{C}$ with the longest possible powder exposure period. The fabrication parameters in the SLS process are mainly dependent on the properties of powder used, so changes in fabrication parameters as the powder ages, gives consistent and good quality fabricated SLS specimens.

As the powder degrades due to exposure to temperature for an extended period through the SLS system, the quality and surface finish of the fabricated SLS specimens become poor. Therefore the amount of virgin power to be added to achieve the target melt index range has varied from $25 \%$ to $42 \%$ depending on the length of time the powder was exposed to a temperature. The melt flow rate index has proved to be a good indicator of powder fitness.

\section{References}

1. Pham DT, Dimov SS (2003) Rapid Prototyping and Rapid Tooling - the key enablers for rapid manufacturing. J Mech Eng Sci 217: 1-23.

2. Kumar V, Dutta D (1997)An assessment of data formats for layer manufacturing Adv Eng Softw 28: 151- 164.

3. Yusuf WAY (2007) An investigation of the Orange Peel Phenomenon. Cardiff University, Wales, UK.

4. Kruth JP, Mercelis P, Vaerenbergh J, Froyen L, Rombouts M (2005) Binding Mechanisms in Selective Laser Sintering and Selective Laser Melting. Rapid Prototyping Journal 11: 26-36.

5. Cowie JMG (1973) Polymer: Chemistry \& Physics of Modern Materials Guildford: Billing.

6. Crawford RJ (1987) Plastics Engineering. 2nd edition, Oxford: Pergamon Press.

7. Gibson I, Shi D (1997) Material Properties and Fabrication Parameters in Selective Laser Sintering Process. Rapid Prototyping Journal 3: 129-136.

8. Mangonon PL (1999) The Principles of Materials Selection for Engineering Design. Prentice Hall, New Jersey, USA.

9. Kohan MI (1995) Nylon Plastics Handbook. Hanser, New York.

10. Beamam JB, Barlow JW, Bourell DL, Crawford RH, Marcus HL (1997) Solid Freeform Fabrication: A New Direction in Manufacturing. Kluwer Academic Publishers, Dordrecht, Netherlands.

11. Brydson J (1999) Plastics Materials. 7th edition, Oxford: ButterworthHeinemann, Massachusetts, USA.

12. Young RJ (1981) Introduction to Polymers. Chapman and Hall, London, UK. 